Estudios Constitucionales, Año 16, No 1, 2018 pp. 15-38

ISSN 07180195

Centro de Estudios Constitucionales de Chile Universidad de Talca

"El dilema presidencialismo vs. parlamentarismo en

América Latina. Apuntes sobre la realidad en el siglo XXI"

Carlos Manuel Villabella Armengol

\title{
EL DILEMA PRESIDENCIALISMO VS. PARLAMENTARISMO EN AMÉRICA LATINA. APUNTES SOBRE LA REALIDAD EN EL SIGLO XXI*
}

\author{
LATIN AMERICA: PRESIDENTIALISM VS. PARLIAMENTARISM. \\ FACTS ABOUT THE DILEMMA IN THE 21ST CENTURY
}

Dr. Carlos Manuel Villabella Armengol Instituto Tecnológico y de Estudios Superiores de Monterrey, México**

RESUMEN: En las últimas décadas del siglo pasado se inicia en América Latina un proceso de reinstitucionalización y redemocratización que tuvo como uno de sus ejes la revisión critica de la forma de gobierno presidencial, en la consideración de que ésta era la causante de las rupturas democráticas y las crisis de gobernabilidad. Del debate, se pasó a la reingeniería del sistema, lo cual se legitimó en las constituciones que se promulgaron en esos años. El presente estudio reconstruye la remodelación del régimen presidencial en la región y valora su resultado, empleando el metaanálisis y el derecho comparado. Se comentan en particular el modelo mexicano porque su Constitución, que cumplió en el 2017 cien años de vigencia, no siguió la tendencia reformadora del resto de los países del área.

SUMMARY: In the last decades of the past century, a process of reinstitutionalization and redemocratization began in Latin America. Which had as one of its axes, the critical revision of the form of presidential government, given that this was prone to democratic ruptures and governance crisis. As a result of the debate, the system was re-established, becoming legitimate in the constitutions promulgated in those years. The present study reconstructs the remodeling of the presidential regime in the region and evaluates its result, using meta-analysis and Comparative Law. In particular, the Mexican model is analyzed, given that its Constitution whose centennial in 2017, did not follow the reform trend of the rest of the countries in the area.

PALABRAS clave: Parlamentarismo vs. presidencialismo, presidencialismo Latinoamericano, forma de gobierno en América Latina, Centenario de la Constitución mexicana.

KEYWORDS: Parliamentarism vs. presidentialism, Latin-American presidentialism, form of government in Latin America, Centenary of the Constitution of Mexico, Mexican presidentialism.

\footnotetext{
* Artículo recibido el 6 de noviembre de 2016 y aprobado el 11 de abril de 2018.

${ }^{* *}$ Profesor del Departamento de Derecho de la Escuela de Ciencias Sociales y Gobierno del Instituto Tecnológico y de Estudios Superiores de Monterrey, Puebla. Director de la Revista IUS (México). Miembro del Sistema Nacional de Investigadores de México.
} 


\section{PRELIMINAR: SiNOPSIS DEL}

\section{CONSTITUCIONALISMO LATINOAMERICANO CONTEMPORÁNEO}

En la obra "Estudios sobre un nuevo constitucionalismo latinoamericano" publicada por Tirant lo Blanch en el 2012, ${ }^{1}$ sostuve que hasta finales de los años setenta de la centuria anterior el constitucionalismo de América Latina se encontraba en impasse, en tanto continuaba reproduciendo el modelo constitucional liberal decimonónico, no había introducido efectivamente los caracteres del Estado social y permanecía al margen de los grandes problemas sociales, económicos y políticos de la región. Era, hasta ese momento, un constitucionalismo nominal, elitista y conservador. Fue en la década de los ochenta que comenzaron a operarse renovaciones institucionales y normativas que rompieron el inmovilismo que señalo.

El primer momento lo marcaron los gobiernos militares de algunos países que, en su ocaso, impulsaron la redacción de nuevas constituciones como instrumento para la apertura democrática. De esa coyuntura emanaron los textos de Chile (1980), Honduras (1982), El Salvador (1983) y Guatemala (1985), que presentaron los siguientes rasgos destacables: incluyeron artículos dedicados a la regulación del régimen económico; incorporaron derechos sociales; algunas introdujeron la figura del Ombusdman como defensor de los derechos humanos; refrendaron cortes constitucionales y salas especializadas (con ello comenzaba a producirse un giro en el modelo de justicia constitucional existente); y ciertos textos insertaron elementos que formalmente mitigaban la preponderancia de la figura presidencial, iniciando así un proceso de transformación en la forma de gobierno que progresaría en los siguientes momentos constitucionales como explicaremos ut infra.

Con la siguiente oleada de cartas magnas: Nicaragua (1987), Brasil (1988), Colombia (1991), Ecuador (1988), Paraguay (1992), Perú (1993) y Argentina (1994), se inició una etapa caracterizada por la adopción de caracteres del derecho constitucional europeo, asumidos con la intención de modernizar la complexión de los documentos constitucionales e incidir en la estabilidad democrática y la vigencia de los derechos humanos. Determinados injertos normativos e instituciones importadas, resultaron inoperantes por no ajustarse al contexto cultural y provocar tensiones que obstaculizaron su implantación, empero, el momento

1 Villabella (2012), pp. 51-77. 
significó el inicio de un nuevo constitucionalismo ${ }^{2}$. Como apuntan Viciano Pastor y Martínez Dalmau: "se anunciaba el fin de una era constitucional nominalista y poco original y del inicio de nuevas luces en el constitucionalismo latinoamericano [...] cambios que pronosticaban la conciliación entre constituciones formales y materiales"3. De éstas, resaltan los documentos de Brasil, Colombia y Ecuador, por las novedades que introdujeron y la proyección social de sus postulados.

Algunos elementos que trazaron nuevas tendencias en el entorno, fueron los siguientes: incorporación de normas de principios y teleológicas; refrendo de vías de participación directa en el poder público; reconocimiento de las minorías étnicas y pueblos originarios, comprometiéndose el Estado con la protección de su identidad cultural y costumbres; regulación profusa de derechos sociales y económicos, a la par que se estipulaba la adopción de políticas públicas para su implementación; legitimación de derechos colectivos y difusos; reconocimiento del rango constitucional de los tratados y convenios suscritos en materia de derechos humanos; e institucionalización de cortes constitucionales y salas especializadas (se configuró un modelo mixto de justicia constitucional).

En el mapa constitucional del continente hay que mencionar finalmente a las constituciones de Venezuela (1999), Ecuador (2008) y Bolivia (2009), que contornaron lo que he identificado como novísimo constitucionalismo, ${ }^{4}$ término con el que significo que desarrollaron aspectos del momento anterior, pero añadieron otros elementos innovadores. Los textos citados fueron elaborados en procesos constituyentes originarios, tuvieron una gran proyección social, innovaron instituciones, y expresaron un talante rupturista con los cánones provenientes del constitucionalismo liberal.

Algunas características que resaltan son las siguientes: alta presencia de principios, valores y fines que proporcionan un plexo axiológico al texto; profuso refrendo de derechos, regulación original de éstos y consagración de figuras novedosas; amplia protección de las minorías étnicas y los grupos originarios, al paralelo del reconocimiento de sus derechos, formas de convivencia y modalidades de autogobierno; empleo de lenguas nativas (aymara, guaraní y quechua);

2 Un sector de la teoría ha utilizado el término nuevo constitucionalismo latinoamericano para referirse en particular al canon que iniciaron las cartas magnas de Brasil (1988), Ecuador (1988) y Colombia (1991), y desarrollaron los textos de Venezuela (1999), Ecuador (2008) y Bolivia (2009). Ver: Viciano y MarTínez (2011), pp. 1-24.

3 Viciano y Martínez (2011), p. 11.

4 Villabella (2014). 
estructuración del poder político público sobre principios organizacionales de nuevo tipo; refrendo de numerosas vías de participación directa que impulsan el empoderamiento ciudadano; reconocimiento del protagonismo del Estado en el logro de objetivos sociales; regulación detallada del régimen económico y el papel del Estado en el manejo de las políticas económicas y financieras; consagración de políticas de integración regional; reconocimiento de la participación del pueblo en el procedimiento de reforma constitucional.

Como se observa, con excepción de México (1917), Costa Rica (1949), Uruguay (1966) y Panamá (1972), el resto de los países de América Latina renovaron sus documentos constitucionales durante las últimas décadas y, aunque en algunos casos las mutaciones fueron más significativas que en otros, se gestó una nueva época constitucional que tuvo, entre sus ejes céntricos, proveer mayor estabilidad política e institucional.

A continuación, repaso la remodelación del régimen presidencial en la región y valoro su resultado, empleando el metaanálisis y el derecho comparado.

\section{LA FORMA DE GOBIERNO DE LA REGIÓN EN ENTREDICHO}

En los años ochenta de la centuria anterior las juntas castrenses y caudillos militares cedieron el poder obligados por las circunstancias y el cambio de estrategia en la Doctrina de Seguridad Nacional implementada por Estados Unidos. Así, en esa década, se organizaron elecciones y se desarrollaron asambleas constituyentes en algunos países.

En ese contexto de recuperación democrática e institucional, se suscitó el debate sobre el funcionamiento de la firma de gobierno presidencial y su incidencia en la democracia y la estabilidad política. El punto de partida del análisis lo constituyó el evento sobre reforma política y estabilidad democrática efectuado en marzo de 1987 en Fortín de Santa Rosa, Uruguay. De las reflexiones del cónclave, emergieron dos escritos que marcaron pauta: en 1985 Juan Linz difundió el trabajo "Presidential and Parliamentary Government. Does it Make a Difference?"5, y en 1988 Dieter Nohlen y Aldo Solari publicaron "Reforma política y consolidación

5 En 1990 se publicó una versión en castellano del artículo en una obra coordinada por Óscar Godoy. Ver: Linz $(1990, a)$. En ese año aparecieron otros dos escritos de este autor en la misma línea, publicados en Journal of Democracy (1990, b, c). 
democrática. Europa y América Latina"6. A partir de ese momento, el tema se convirtió en centro de las discusiones teóricas.

Un sector de los teóricos apostilló que el presidencialismo era un modelo de gobierno que, por su estructura orgánica rígida, tendía a la ingobernabilidad, los bajos rendimientos democráticos y la parálisis funcional; propiciando que las crisis de gobernabilidad desembocaran en la desestabilización del régimen político en su conjunto:

Una comparación cuidadosa entre parlamentarismo y presidencialismo en cuanto tales, lleva a la conclusión que, en balance, el primero es más conducente hacia democracias estables que el último. El historial de los países gobernados por un presidente varía de malo a desalentador y nos lleva a preguntarnos si su problema político no se debe al propio presidencialismo ${ }^{7}$.

Otro autores como Nohlen fueron comedidos, ${ }^{8}$ razonando que no existen tipologías ideales de formas de gobierno y que el análisis dicotómico presidencialismo $v s$. parlamentarismo basado en el diagrama institucional es engañoso, porque desconoce el medio ambiente social, cultural, económico y político en que se desempeña el sistema de gobierno, lo cual constituye un factor determinante de su funcionalidad. Se obvia además que el parlamentarismo no funciona con la misma eficiencia en toda Europa y que el presidencialismo tiene estabilidad en determinados países: ${ }^{9}$

Debatir, por tanto, sobre las bondades de los sistemas de gobierno en el nivel de los tipos ideales conduce a una grave deformación teórica y práctica. Se sabe que los tipos ideales como tales no existen en forma pura ni son «buenos» ni «malos» por su correspondiente conformación. Constituyen un marco de características en el cual se agrupa la gran multiplicidad de sistemas concretos. Por tanto, el desarrollo de las formas de gobierno se explica a partir de situaciones históricas específicas ${ }^{10}$.

A partir de estas valoraciones, se delinearon diversas alternativas. Verbigratia, Linz defendió la adopción de la forma de gobierno parlamentaria, ${ }^{11}$ Nohlen apreció

${ }^{6}$ Nohlen y Solari (1988).

7 Linz y Valenzuela (2013), p. 12.

8 Nohlen (1991 a, b), (2012), (2013).

9 En este sentido ver también: Orozco (2008): pp. 793-858.

10 Nohlen (1998): pp. 71, 73.

11 LINZ (1990, a), pp. 60 y ss. 
la necesidad de introducir elementos de este sistema, ${ }^{12}$ Nogueira contempló la posibilidad de instrumentar un régimen semiparlamentarismo, ${ }^{13}$ y Sartori postuló una tipología de presidencialismo alternativo o de dos motores ${ }^{14}$.

El debate repercutió en los procesos constituyentes que se sucedieron en los siguientes años y se convirtió en tema central de las reformas políticas efectuadas en la región.

\section{Del Debate a la METAMorfosis Del PRESidenCialismo}

En América Latina todos los países adoptaron la forma de gobierno presidencial tras su independencia, con la excepción de México y Brasil que instauraron un régimen monárquico. En México existió un Primer Imperio de 1821 a 1823 que se extendió a Centroamérica, y luego un Segundo Imperio entre 1864 y 1867. En Brasil la experiencia monárquica se extendió de 1825 a 1889. Es de citar también a Uruguay que tuvo un ensayo de gobierno convencional entre 1917 y 1933 , al que siguió un régimen semiparlamentario instaurado por la Constitución de 1934, y luego, un segundo período de forma de gobierno directoral entre 1952 y 196715 .

La recepción y predominio del presidencialismo se ha explicado con argumentos históricos, culturales y de gobernabilidad, pero una razón puntual fue el influjo de Estados Unidos. No obstante, su refrendo no constituyó un clon del prototipo norteamericano, ni su funcionamiento ha gozado de la misma estabilidad. Ejemplo de lo primero es la regulación más detallada del funcionamiento y facultades del ejecutivo y el legislativo, la elección directa del presidente, la mayor incidencia de éste en el proceso legislativo, la ausencia de vicepresidente en algunos países o la existencia de dos en otros; etc. Expresión de lo segundo es la fragilidad institucional del sistema en Latinoamérica, la dificultad para generar democracias estables, y su ejercicio caudillista, clientelar y populista.

La redemocratización ocurrida durante las últimas décadas del siglo anterior impulsó la revisión crítica del diseño orgánico-funcional del sistema, como se mencionó ${ }^{16}$. Las acciones de reforma provocaron la mutación de la forma de go-

12 Nohlen (1991, b): pp. 20 y ss.

13 Nogueira (2008): p. 769.

14 Giovanni (1996): p. 168.

15 Gross (1978). Ratto (2005): pp. 35-75.

16 El cambio de escenario político e institucional en América Latina estimuló los estudios sobre calidad de la democracia en la región. Ver entre otros: Molina (2000). Carpizo (2007). Cameron (2010), pp. 5-20. 
bierno en dos puntos cardinales: delineación de un ejecutivo menos hegemónico e introducción de instituciones parlamentarias. En esa tesitura, las mutaciones fueron de una soft dimension (conversión nominal de los secretarios en ministros, afirmación de que el poder ejecutivo se desempeña por el presidente y los ministros) a una strong dimension (introducción de órganos de gobierno, refrendo del mecanismo de censura a los ministros).

Aunque las reformas comenzaron en las constituciones redactadas en los años sesenta del siglo anterior, los diferentes momentos constitucionales descritos en el primer epígrafe implicaron momentos de profundización en la conversión del sistema.

La gradación del presidencialismo en el área fue advertida por estudiosos del derecho comparado. Verbigratia, Sánchez Agesta distinguió entre presidencialismo puro, atenuado y con aproximación al parlamentarismo ${ }^{17}$. Colomer Vidal diferenció el presidencialismo hegemónico de excepción, hegemónico constitucional democrático, de sujeción parlamentaria y de equilibrio de poderes ${ }^{18}$. Loewenstein destacó al presidencialismo puro, atenuado y de aproximación al parlamentarismo ${ }^{19}$. Bidart Campos apreció el presidencialismo puro, híbrido e intermedio ${ }^{20}$. Biscarretti di Ruffia calificó el presidencialismo en democrático y autoritario $^{21}$. Nogueira Alcalá consideró el presidencialismo puro, atenuado y parlamentarizado ${ }^{22}$. Carpizo identificó el presidencialismo puro, predominante, atemperado, con matices parlamentarios y parlamentarizado 23 .

Siguiendo esta línea de pensamiento, pero simplificando las categorías, distingo en el mapa constitucional actual de Latinoamérica tres tipologías de presidencialismo: puro, atenuado y con correctivos parlamentarios.

El modelo puro tiene una fisonomía y funcionamiento cercano al original arquetipo norteamericano, y está presente en Chile y México. Debe anotarse

\footnotetext{
André, pp. 125-154. Corral (2011). Levine y Molina (2011), pp. 95-113. Vargas (2011, pp. 67-94. MASSAL (2012), pp. 29-45.

17 SÁNCHeZ (1980), pp. 245 y 246.

18 Colomer (1992) pp. 139 y ss.

19 Loewenstein (1998), pp. 42 y ss.

20 Bidart (1988), pp. 54 y ss.

21 Biscarettidi (2000), pp. 190 y ss.

22 Nogueira (2008), p. 726.

23 Carpizo (2007), pp. 40-42.
} 
como diferencia, que la Constitución chilena dedica una sección a los ministros de estado que otorga un mayor realce a estos funcionarios, aunque el texto precisa que son colaboradores directos e inmediatos del presidente en el gobierno y la administración del Estado.

El modelo atenuado se caracteriza por la despersonalización del poder ejecutivo y la existencia de un órgano de gobierno, aspectos que transmutan el diseño primigenio del sistema: Se ha contornado en Brasil, Costa Rica, El Salvador, Honduras, y Nicaragua:

- Las cartas magnas de Brasil, Costa Rica y El Salvador exponen que el poder ejecutivo es desempeñado por el presidente y los ministros como colaboradores; o como señala el texto salvadoreño: por un órgano ejecutivo integrado por el presidente, el vicepresidente, los ministros y los viceministros.

- Costa Rica, El Salvador, Honduras y Nicaragua institucionalizaron consejos de ministros encabezados por el presidente (Costa Rica lo denomina consejo de gobierno). La constitución de los primeros dos países le reconoce funciones al órgano y le dedica varios artículos a su regulación.

- En estos países se reconoce al legislativo la facultad de solicitar informes a los ministros e interpelarlos. La Constitución de Brasil plantea que el congreso fiscaliza los actos del ejecutivo, y la de El Salvador que puede recomendar al presidente la destitución de algún ministro.

El modelo de presidencialismo con correctivos parlamentarios se tipifica por la legitimación de instituciones de gobierno y mecanismos funcionales del sistema parlamentario, aunque no hay rasgos homogéneos en los países que catalogamos de esta forma. Se ha configurado en Argentina, Bolivia, Colombia, Ecuador, Guatemala, Panamá, Paraguay, Perú, Uruguay y Venezuela:

- En Bolivia, Guatemala y Panamá el poder ejecutivo se deposita en un órgano integrado por el presidente, el vicepresidente y los ministros. En las constituciones de Ecuador y Venezuela se señala que el presidente es jefe de Estado y de gobierno, pero al paralelo, éste ejerce la función ejecutiva junto a los ministros y funcionarios. Se exceptúan los textos de Argentina y Colombia que realzan la figura presidencial al destacar que es jefe de Estado, de gobierno, y suprema autoridad administrativa.

- Con excepción de Ecuador, los países señalados institucionalizan un órgano de gobierno. En Argentina se denomina gabinete de ministros, en Panamá consejo de gabinete, en Colombia gobierno nacional, y en Bolivia, Guatemala, Paraguay, Perú y Uruguay, consejo de ministros. 
- En todos los casos al órgano de gobierno se le reconocen atribuciones propias. El texto constitucional de Argentina refrenda solo las funciones del jefe de gabinete, el de Bolivia las atribuciones de los ministros, y el de Venezuela precisa las facultades que el presidente debe ejercer en compañía del consejo de ministros. Resalta la Constitución de Uruguay que no reconoce facultades al presidente de manera unipersonal, sino junto a los ministros.

- Se refrenda el voto de censura o procedimiento de confianza contra los ministros, procedimiento que, de prosperar, los obliga a dimitir. En Uruguay la censura puede ser individual, plural contra varios ministros y colectiva contra el consejo en pleno. En Venezuela la censura procede también contra el vicepresidente.

- En Argentina y Perú se legitima la figura del primer ministro que coordina la actividad administrativa, dirige el funcionamiento del consejo y representa al gobierno ante el legislativo. El jefe de gabinete argentino tiene responsabilidad política directa ante el congreso. El presidente del consejo de ministros peruano tiene que presentar al congreso el programa político y solicitar su confianza.

- En Perú y Uruguay se regula la cuestión de confianza, mecanismo por el cual el gobierno solicita al legislativo una votación que exponga el respaldo que tiene para instrumentar determinada política.

- Los documentos constitucionales de Ecuador, Perú, Uruguay y Venezuela legitiman que el presidente puede disolver al legislativo en determinados supuestos.

Como se advierte, las variables organizacionales y funcionales que se introdujeron en la forma de gobierno de América Latina implicaron una mutación de éste, acercándolo formalmente al modelo parlamentario, sobre todo en las naciones reseñadas en el último grupo.

\section{4. ¿'SE LOGRARON LOS PROPÓSITOS?}

Un sector de la academia con el que comulgué, ponderó el derecho constitucional gestado durante las últimas décadas del siglo anterior por su vocación transformadora, sus propuestas experimentales y su ruptura con las fórmulas del constitucionalismo de raigambre liberal. Luego de tanto años de inmovilismo y de calco de tendencias foráneas, un movimiento constitucional de cariz iconoclasta, innovador y procurador de justicia social, provocaba entusiasmo. En un artículo publicado en el Boletín Mexicano de Derecho Comparado señalé que, no obstante ello, la exuberancia de principios y contenidos de los textos constitucionales de última data, la fragilidad de algunas de sus propuestas institucionales, su depen- 
dencia de una economía con superávit, y su filiación con un liderazgo populista incapaz de reciclarse, constituían aspectos que atentaban contra su continuidad ${ }^{24}$.

Un análisis crítico que enjuicie en qué medida se transfiguró realmente la realidad constitucional latinoamericana se encuentra pendiente, y en esa tesitura, igualmente el cuestionamiento de si la metamorfosis a la forma de gobierno presidencial logró el propósito deseado. Sobre este último punto, externo tres evidencias que lo ponen en duda.

La primera es que, durante los últimos años, en más de veinte ocasiones, se ha interrumpido el desempeño del poder ejecutivo y el legislativo, por acciones sociales, golpe militar o deposición anticipada. Ello refleja que los cambios jurídicoinstitucionales no impidieron la fragilidad del desempeño del poder político y la intromisión de las Fuerzas Armadas en la vida pública. Este dato es inadvertido en ocasiones porque resulta menor en comparación con la historia precedente del continente, donde se produjeron más de 100 golpes de Estado, aproximadamente en 130 ocasiones hubo presidentes interinos porque los titulares no culminaron su mandato, y en más de veinte de ocasiones gobernaron juntas militares que llegaron al poder por medios violentos ${ }^{25}$.

Enumero los momentos en se ha quebrantado la continuidad de los poderes públicos durante los últimos años: destitución de Fernando Collor de Mello en Brasil (1992) mediante juicio político; remoción de Carlos Andrés Pérez en Venezuela (1993) por el poder legislativo; separación de Abdala Bucarán Ortiz en Ecuador (1997) por el órgano legislativo; renuncia de Raúl Cubas Grau en Paraguay (1999) ante la amenaza de juicio político; separación de Alberto Fujimori en Perú (2000) por parte del congreso; derrocamiento de Jamil Mahuad Witt en Ecuador (2000) por un movimiento indígena-campesino que logró el apoyo de las Fuerzas Armadas; dimisión de Fernando de la Rúa en Argentina (2001) ante la presión de una rebelión popular que sumó el apoyo de la clase media de Buenos Aires; golpe de estado a Hugo Chavez Frías en Venezuela (2002), malogrado por el movimiento popular que obligó al Ejército a liberarlo; expulsión de Gonzalo Sánchez de Losada de Bolivia (2003) por un movimiento popular; destronamiento de Lucio Gutiérrez Borbúa de Ecuador (2005) por un movimiento cívico-militar; renuncia de Carlos Mesa Gisper en Bolivia (2005) compulsado por una crisis social y la huelga liderada por la Central Obrera Boliviana; golpe de Estado a

24 Villabella (2017), pp. 943-979.

25 Sobre la militarización de la política en el continente ver: SERRANO (2010), pp. 175-183. 
José Manuel Zelaya Rosales en Honduras (2009); separación de Fernando Lugo Méndez de Paraguay (2012) mediante impeachment express; abdicación de Otto Pérez Molina en Guatemala (2015) al ser desaforado por acusaciones de corrupción; dimisión de Dilma Vana da Silva Ruseff en Brasil (2016) por juicio político (debe diferenciar el impeachment que se realizó a Collor de Mello por acusaciones de corrupción y tráfico de influencia, de los efectuados a Fernando Lugo y Dilma Ruseff que tuvieron las características de un golpe de estado jurídico).

En esta enumeración hay que incluir los autogolpes de Estado efectuados por Alberto Fujimori en Perú (1992) y Jorge Serrano Elías en Guatemala (1993), que interrumpieron el mandato del poder legislativo. Fujimori, con el respaldo del Ejército y la simpatía de parte de la población, disolvió el congreso e intervino el poder judicial. Serrano disolvió el legislativo, la corte constitucional, la corte suprema y destituyó al procurador, pero luego de varios días de crisis abandonó la presidencia.

El segundo dato que reseño es que las facultades jurídicas y meta constitucionales del presidente no mermaron en la práctica, en algunos países incluso, se ampliaron mediante reformas constitucionales. Ello es ostensible, por ejemplo, en la intervención del titular del ejecutivo en la formación de las leyes. En este punto, cuenta con iniciativa legislativa, incluso, exclusiva en determinadas materias (Chile, Brasil, Colombia y Ecuador); tiene facultad para solicitar trámite de urgencia para sus proyectos legislativos (Chile, México, Brasil, Nicaragua, Bolivia, Colombia, Ecuador y Paraguay); puede emitir normas con fuerza de ley previa habilitación (Chile, Brasil, Argentina, Colombia, Panamá, Perú y Venezuela); cuenta con poder de veto legislativo total y parcial superable solo por mayoría extraordinaria de las cámaras.

El predominio del ejecutivo se manifiesta también en la potestad que tiene éste para incidir en nombramientos, algunos, trascendentes en el contrapeso de poderes como los magistrados de la máxima instancia judicial o los jueces del órgano de control constitucional (México, Panamá, Guatemala, Nicaragua, Chile, Argentina, Brasil, Ecuador, Bolivia, Venezuela). Asimismo, su facultad para declarar estados excepcionales en los que se asumen poderes extraordinarios o restringen derechos (Honduras, Chile, Argentina, Paraguay, Venezuela, Bolivia, Perú).

El tercer aspecto que destaco es la reelección presidencial, aspecto que ha progresado en los últimos años, y en el que se produjo una traslación de postura, ya que, de la prohibición o aceptación excepcional, se ha franqueado la reelección inmediata e ilimitada. 
A tenor de los cambios constitucionales y electorales producidos en las últimas décadas, se distinguen cuatro fórmulas: ${ }^{26}$ la reelección inmediata, que puede ser cerrada si el presidente no puede volver a postularse luego del siguiente mandato, o abierta si no tiene límites (Argentina, Brasil, Honduras); la reelección alterna, cerrada o abierta (Chile, Costa Rica, El Salvador, Panamá, Perú, Uruguay); la reelección indefinida (Bolivia, Ecuador, Nicaragua, Venezuela); y la no reelección (Colombia, Guatemala, México, Paraguay).

La tendencia continuista comenzó en los años noventa con los presidentes Alberto Fujimori de Perú, Carlos Menen de Argentina y Fernando Henrique Cardoso de Brasil, en un ambiente de resultados económicos positivos. Posteriormente, continuó desarrollándose tanto por gobiernos de derecha como de izquierda ${ }^{27}$. El continuismo fue legitimado mediante el referendo (Venezuela), la reforma de la Carta Magna por el legislativo (Brasil, Perú, Ecuador) y la interpretación del órgano de control constitucional (Bolivia, Honduras, Costa Rica, Nicaragua). De estas variantes, la consulta al constituyente originario resultó la vía más plausible por la legitimidad intrínseca que posee éste para cambiar la regla de reconocimiento de un ordenamiento jurídico.

Cerrando estas líneas se contabilizaba el resultado del referendo efectuado en Ecuador en el mes de febrero que, entre las preguntas que realizó, inquiría sobre la abrogación de la reelección indefinida, indicando los resultados que ganó la opción de su eliminación. En la región existen dos antecedentes al respecto: en Venezuela en el 2007, un plebiscito rechazó la reelección, aunque luego fue aprobado por otra consulta popular en febrero del 2009; en Bolivia en febrero del 2016, un referendo denegó la posibilidad de reelección consecutiva, sin embargo, una sentencia del tribunal constitucional de noviembre del 2017 la legitimó.

Caso sui generis en este tema, lo constituye Colombia porque migró de una postura a otra. La Constitución de 1991 contempló la no reelección en el artículo 197, pero en noviembre de 2004 el congreso aprobó una enmienda que la legitimó, lo cual fue ratificado por la corte constitucional un año después. En el 2010 este órgano rechazó una iniciativa de referendo que pretendía reformar de nuevo el texto constitucional a fin de permitir una segunda reelección directa del

26 Ver: Negretto (2009), pp. 39-54.

27 En lo últimos años se han realizado estudios empíricos que vinculan el éxito de la iniciativa reeleccionista con la situación económica del momento, el apoyo popular que existía a la gestión presidencial, el liderazgo que tenía el presidente en su partido, etc. Ver entre otros: Aquino (2012), pp. 247-279. Penfold, et al., (2014), pp. 537-559. TREMinio (2015), pp. 147-173. 
presidente. En junio de 2015 el congreso derogó el cambio del 2004 y sancionó la no reelección.

El tema de la reelección presidencial impacta el equilibrio de poderes y la democracia, tema que ha suscitado diversos estudios en la última década ${ }^{28}$. La reelección se ha criticado arguyendo que degrada la democracia, induce la personalización del poder, desequilibra la lid electoral al proporcionar ventajas a uno de los contendientes y obstaculiza el advenimiento de nuevas figuras políticas. También se ha lisonjeado alegando que concreta la libertad de sufragio pasivo, constituye una vía para premiar el buen gobierno, posibilita que se desplieguen políticas que por su magnitud no se alcanzan en un período corto, y es una manera de no desaprovechar el capital político de un presidente con ascendencia.

Por su parte, la reelección inmediata es censurada porque supone una ventaja para el presidente/candidato que aprovecha su visibilidad pública, y aprovecha además la maquinaria estatal y los recursos públicos. La opción de reelección indefinida es duramente enjuiciada porque significa un quiebre de la alternabilidad en el poder, hipertrofia la figura presidencial, propende el cesarismo y le impregna rasgos autoritarios al régimen: "Puede ser la consagración a nivel jurídico-constitucional de un régimen que de hecho es ya una dictadura, o bien coadyuvar a que un régimen de contornos democráticos se deslice con mayor facilidad hacia otro de corte dudosamente democrático (...) Presidencialismo fuerte y reelección indefinida se refuerzan mutuamente" 29.

El reeleccionismo presidencial es un fenómeno en evolución y su impacto en la calidad de la democracia necesita de estudios empíricos que interconecten diversas variables (duración del mandato presidencial, facultades del presidente, controles del legislativo, fortaleza del sistema de partidos, atrofia del diagrama institucional, etc.) y valoren su consecuencia para la institucionalidad y estabilidad democrática de Latinoamérica. No obstante, es una variable que, sin lugar a dudas, refuerza la figura del jefe de Estado:

(...) cabe plantearse la cuestión de cuáles son las implicaciones que tienen estas reformas en los sistemas políticos latinoamericanos y si estas acciones plantean un nuevo matiz al nivel del régimen presidencialista para la región. El nuevo legado

28 Ver entre otros: CARey (2003), pp. 119-133. Cheresky (2006), pp. 14-26. Buquet (2007), pp. 35-49. Arenas y Valencia (2009), pp. 77-96. Mayorga (2008). McConnell (2010), pp. 74-80. Martínez y Barahona (2012), "pp. 109-136. ACUÑa (2015), pp. 73-87.

29 Serrafero (2011), pp. 253, 256. 
plantea la necesidad de nuevas interpretaciones desde la política comparada de los sistemas políticos en la región, que se han ido decantando a favor de la continuidad, pese a la herencia dictatorial de un pasado reciente; por lo tanto, cabe la necesidad de examinar nuevas premisas que superen la excesiva carga de críticas normativas a la reelección y se aboquen a aportar explicaciones que permitan comprender mejor las nuevas transformaciones en esta materia ${ }^{30}$.

\section{EsTUdio DE CASO: El MODELO PRESIDENCIAL MEXICANO EN SU CENTENARIO}

La Constitución mexicana de 1917 constituye un documento excepcional en América Latina por su vigencia centenaria que sobresale en un entorno de extrema fragilidad constitucional. Descuella históricamente también, por la regulación precursora de derechos laborales (derecho al trabajo, jornada máxima de ocho horas, protección al trabajo femenino y juvenil, derecho al descanso, protección a la maternidad, salario mínimo, salario igual por trabajo igual, protección en caso de accidente de trabajo o enfermedad profesional, derecho a la huelga, indemnización en caso de despido) y la innovación del juicio de amparo como mecanismo de garantía de los derechos.

En ese lapso, el texto ha tenido más de 700 enmiendas, cuestión que la convierte en el documento constitucional más reformado del mundo, dato que también la distingue ${ }^{31}$. No se han modificado 22 artículos, representativos del $6 \%$ del texto original aproximadamente. Esta cantidad de reformas parciales ha provocado artículos extensos que yuxtaponen contenidos de manera inorgánica. A ello debe añadirse, que los cambios formales no siempre estuvieron acompañados de las políticas públicas necesarias para instrumentarlo.

Ante este hecho, sobreviene la siguiente pregunta: por qué luego de cien años de vigencia de la Constitución, pero, sobre todo, de los cientos de reformas que la transfiguraron, no ha progresado la idea de redactar un nuevo texto que exprese la renovación del pacto constituyente y sistematice coherentemente las reformas introducidas. La interrogante es respondida a priori, señalando que en México no hubo interrupciones en la vida política e institucional del país, lo que posibilitó que la Constitución perdurara. Sin embargo, aunque la afirmación es cierta,

\footnotetext{
30 Treminio (2013), p. 82.

31 Las reformas se han clasificado en formales, innovadoras, relacionadas con los poderes públicos, federalizadoras y seguidoras de tendencias. Fix-Zamudio y Valencia (2011), pp. 119 y ss. Una relación de las reformas efectuadas a la Constitución mexicana puede encontrarse en: Centenario Constitución Política de los Estados Unidos Mexicanos, Reformas a la Constitución de 1917, Cámara de Diputados, consultado 14 de agosto de 2016, disponible en: http://www.diputados.gob.mx/LeyesBiblio/ref/cpeum_per.htm.
} 
debe ser acotada con dos razones que la matizan, una cultural y otra política. En ese sentido, es elocuente el razonamiento de Fix-Zamudio y Valencia Carmona que trasluce el peso monumental que tiene la Constitución de Querétaro en la sociología jurídica y el imaginario nacional:

Su carga histórica es tan grande que en la Constitución los mexicanos recrean la herencia ideológica de nuestros sacudimientos sociales, los avances que logró el movimiento revolucionario de este siglo y los principios que rigen a la sociedad civil del presente. A diferencia de otros países, en el nuestro la Constitución ha sido símbolo de estabilidad política y de unidad nacional, en torno a la cual partidos, grupos políticos, y los propios ciudadanos, han desenvuelto las actividades que les son propias. Sentimiento constitucional tan acendrado es difícil de cultivar en los pueblos, por eso hay que mantenerlo y acrecentarlo, tanto porque enraíza en la historia viva, como por su influencia determinante pata nuestro destino común ${ }^{32}$.

Sobre esa idea, la clase política, e incluso, la academia, ha insistido sobre lo innecesario de un nuevo documento constitucional, arguyendo las siguientes razones: se rompería con una historia y tradición constitucional singular; las reformas efectuadas conservan las decisiones políticas trascendentales a la par que introdujeron instituciones novedosas; la idea de una renovación constitucional no representa el sentir general, no resulta prudente políticamente, ni conveniente en época de crisis.

En relación con el tema del estudio, es de resaltar la atipicidad de la forma de gobierno presidencial de México porque no siguió la tendencia del resto de los países que se ha comentado.

La idea de rediseñar el modelo de gobierno tuvo algún realce con posterioridad al 2000, año en que se produjo la alternancia política con el triunfo en la presidencia de la República del Partido Acción Nacional. En esa línea, una de las últimas propuestas de reforma fue valorada en comisiones de la LXI Legislatura, y contempló los siguientes aspectos: control político del legislativo a los funcionarios del Estado, el gobierno y la administración; aprobación del Plan Nacional de Desarrollo y el presupuesto de la federación por ambas cámaras; control sobre informe presidencial; responsabilidad política del presidente y de los altos mandos del poder ejecutivo; posibilidad de sustitución del presidente; limitación del fuero

32 Fix-Zamudio y Valencia (2011), pp. 110-111. 
constitucional; revocación de mandato; legitimación de referéndum constitucional obligatorio y potestativo; adopción de un Consejo de Estado 33 .

Si analizamos los capítulos II y III que establecen los poderes ejecutivo y legislativo, comprobamos que los artículos 50 y 80 que los describen no se han enmendado. A contrario sensu, el resto del articulado se ha modificado en 238 ocasiones (aproximadamente el 35\% del total de las reformas constitucionales), detectándose que algunos de los preceptos se encuentran entre los más transfigurados: el artículo 73 (facultades del Congreso) en setenta y siete ocasiones, el 74 (atribuciones de la Cámara de Diputados) en diecisiete momentos, el 76 (facultades del Senado) y el 79 (estatuye la entidad de fiscalización de la federación) quince veces, y el 89 (funciones del presidente) en dieciocho intervalos.

De las enmiendas efectuadas en estos artículos, merecen destacarse cuatro aspectos relacionados directamente con las funciones del presidente y el control de éstas:

- Regulación de facultades a las cámaras que implican la participación de éstas en decisiones del ejecutivo. En la Cámara de Diputados: ratificar el nombramiento del secretario de hacienda, revisar la cuenta pública y aprobar del plan nacional de desarrollo. En el Senado: aprobar los tratados internacionales que suscribe el presidente, ratificar el nombramiento de los secretarios de estado, designar a los ministros de la Suprema Corte y autorizar la estrategia nacional de seguridad.

- Institucionalización del ente superior de fiscalización con potestad para controlar los ingresos, egresos, manejo, custodia y aplicación de los fondos y recursos del Estado en manos de los diferentes órganos federales y estaduales, lo cual implica un ejercicio de control.

- Reformulación del procedimiento de informe del presidente ante el legislativo. El artículo 69 planteaba en el texto original que, a la apertura de sesiones ordinarias del primer período del congreso asistirá el Presidente de la República y presentará un informe por escrito en el que manifieste el estado general que guarda la administración pública del país. Con la reforma del 2008 desapareció la frase asistirá el presidente, quedando solo la obligación de entregar el informe; añadiéndose un segundo párrafo que plantea que cada cámara analizará el informe y podrá solicitar al presidente ampliar la información mediante pregunta por escrito y citar a los secretarios de Estado.

- Refrendo del trámite de urgencia en la iniciativa de ley del presidente. El artículo 71 señalaba en el texto primigenio, que la iniciativa de las leyes correspondía

33 Comisiones Legislativas, LXI Legislatura, Reforma de Estado, consultado 10 de noviembre de 2015, disponible en: http://www.senado.gob.mx/comisiones/LX_Legislatura/reformadelestado/._ 
al presidente, los diputados, senadores y las legislaturas de los estados. La reforma del 2008 reconoció iniciativa legislativa a los ciudadanos, y agregó un párrafo en el que estampa que al inicio de cada período ordinario el presidente puede presentar hasta dos proyectos para que fueran tramitados con carácter preferente.

A tenor de lo reseñado, Carpizo consideró que se había producido la parlamentarización del sistema de gobierno; ${ }^{34}$ y los ya citados Fix-Zamudio y Valencia Carmona aseguraron que se ha transitado de un "régimen presidencialista a uno simplemente presidencial, debido a que existen varios signos que anuncian un esquema diverso en la organización y funcionamiento del ejecutivo ${ }^{35}$. Por el contrario, Jaime Cárdenas sostuvo que el ajuste del modelo de gobierno en México está realmente pendiente, esbozando como algunas de las directrices a seguir las siguientes: introducir un primer ministro o figura análoga que coordine la acción de gobierno, intensificar los controles políticos al ejecutivo (aprobación de nombramientos y miembros del gabinete, censura a los secretarios, etc.), permitir la reelección del presidente, concederle facultad al ejecutivo de disolver al legislativo en determinados supuestos, etc ${ }^{36}$.

Considero que la forma de gobierno en México no ha cambiado en su esencialidad. El presidente no ha visto disminuida su hegemonía como jefe de Estado y Gobierno, cuenta con imperium metaconstitucional que ejerce en el funcionamiento del aparato público y en la vida interna de su partido político. $A$ sensu contrario, no existen mecanismos de control político efectivo que se puedan ejercer sobre la función de gobierno, cuestión por la que el jefe de Estado constituye un César sexenal inamovible. La remodelación producida en la dinámica del sistema de gobierno tiene que ver con la pérdida de hegemonía política del Partido Revolucionario Institucional, la alternancia efectuada en la presidencia de la República, el surgimiento de nuevas fuerzas políticas, y el cambio de escenario en el Congreso de la República.

El estatismo del presidencialismo mexicano guarda relación con la razón, ya expuesta, de la estabilidad que ha gozado el régimen político, pero influye también que el tema ha sido visto con cautela, diría incluso, con miedo:

(...) un análisis serio, profundo y sistemático, revela que dichas propuestas de reforma -en realidad una contrarreforma nociva para los mejores intereses de la

34 Carpizo (2012), pp. 31 y ss.

35 Fix-Zamudio y Valencia (2011), pp. 835 y ss. En un sentido similar Valadés (1988), p. 409.

36 Cárdenas (1994), pp. 170 y ss. 
República- constituyen un "espejismo" altamente peligroso pues implican nada menos que el desmantelamiento total de la "coraza" o "blindaje" constitucional que brindan al Estado mexicano (...) el original sistema presidencial plasmado en 1917 (...) deben evitarse en los procesos de reingeniería constitucional (...) los procesos de extrapolación e imitación.

\section{Colofón}

El constitucionalismo en América Latina, tras varias décadas de inmovilismo, inició en los años ochenta del siglo pasado una transformación sustancial que se vio reflejada en las cartas magnas que se promulgaron en casi todos los países en ese lapso. En la remodelación constitucional de la región pueden apreciarse dos entornos. El primero, la modernización de los textos a partir de la adopción de elementos neoconstitucionales de raigambre europea. El segundo, la incorporación de aspectos innovadores, contestatarios al derecho liberal, autóctonos, de compromiso social, y que apuestan por que la constitución sea un instrumento de cambio. Por ello, en páginas anteriores hablé de un nuevo constitucionalismo y un novisimo constitucionalismo, nuncupationes habeat que pretende diferenciar ambos esos contornos.

Una invariante de ese proceso fue la mutación de la forma de gobierno presidencial, a partir, fundamentalmente, de la introducción de órganos y mecanismos funcionales del parlamentarismo. La metamorfosis se justificó por la valoración de que el diseño orgánico-funcional del sistema era responsable de las rupturas institucionales y las crisis de gobernabilidad. Se analizaba que el predominio del ejecutivo, por las facultades constitucionales que poseía y el protagonismo extrajurídico que ejercía en los diferentes ámbitos del poder político público, impedía un correcto equilibrio entre poderes, lo que a su vez era responsable de los déficits democráticos. Se revelaban también, los siguientes rasgos que incidían negativamente en su eficiencia: legitimidad plebiscitaria del ejecutivo y legislativo, cuestión que suscita el bloqueo cuando existe bipolaridad en las fuerzas políticas; juego de suma cero que propicia una dinámica política antagónica; falta de liderazgo presidencial cuando éste queda elegido por una minoría menor; doble rol del presidente al reunir los atributos simbólicos del jefe de Estado y encabezar el gobierno y la administración, ambivalencia que origina el desgaste de la figura; ausencia de mecanismos para solucionar los conflictos entre poderes y reordenar las mayorías. 
La ilusión del rediseño fue que, la ingeniería constitucional, per se, mitigaría el hegemonismo presidencial, surtiría estabilidad institucional y mejoraría la calidad democrática. Empero, como expuse ut supra, una valoración prima facie, evidencia que el objetivo fue incumplido: i) perdura la hipertrofia de la figura presidencial porque, a contracorriente del movimiento de conversión del presidencialismo, se introdujeron reformas que fortalecieron al ejecutivo en varios ámbitos, incluido el proceso de creación normativa; ii) no se logra el equilibrio del legislativo con el ejecutivo, entre otros factores porque los mecanismos de control del segundo sobre el primero son, en la mayoría de los casos, formales e insuficientes; iii) la legitimación de la reelección presidencial aristocratiza al presidente y la elite de poder que lo respalda; iv) no se han evitado las rupturas institucionales; v) la presencia militar y el uso inconstitucional de la fuerza pública, continúa siendo una salida a los conflictos de gobernabilidad, tanto desde la izquierda como desde la derecha.

El fortalecimiento del ejecutivo, por tanto, no solo no cambió sustancialmente, sino, además, se reforzó por vía constitucional. Incluso, en un país como México donde no se siguió la tendencia hacia parlamentarización y el modelo de gobierno presidencial permaneció inalterable durante cien años, elementos como el control del legislativo sobre el ejecutivo se ha formalizado aún más, provocando que el presidente funja como César intocable durante seis años.

\section{Bibliografía}

AcuÑa VillarRaga, Fabián Alejandro (2015): “¡Presidentes desatados?: Reelección Presidencial y cambio institucional en el área andina”, Análisis Político, (Vol. 28, No 83), Universidad Nacional de Colombia, pp. 73-87.

André Melo, Marcus (2011): "Accountability, diseño institucional y calidad de la democracia", Revista Latinoamericana de Política Comparada, (Vol. 5, julio), Centro Latinoamericano de Estudios Políticos, pp. 125-154.

AndREa SÁnchez, Francisco José de (2008): "La restauración de los tres pilares del constitucionalismo mexicano: el original sistema presidencial, el principio de no-reelección y el articulado de contenido social”, Boletín Mexicano de Derecho Comparado, (Número conmemorativo, sexagésimo aniversario), UNAM, pp. 1-13.

Aquino R., José Ángel (2012): "Reelección presidencial y equidad en las campañas electorales", Construyendo las condiciones de equidad en los procesos electorales, 
(Costa Rica, Instituto Iberoamericano de Derechos Humanos-Centro de Asesoría y Promoción Electoral), pp. 247-279.

Penfold, Michael; Corrales, Javier y Hernández, Gonzalo (2014): “Los Invencibles: La reelección presidencial y los cambios constitucionales en América Latina”, Revista de Ciencia Política, (Vol. 34, No 3), Instituto de Ciencias Políticas, Universidad Católica de Chile, pp. 537-559.

Arenas, Juan C. y Valencia, Germán D. (2009): "Elecciones y reelecciones presidenciales en América Latina”, Perfil de Coyuntura Económica, (No 13, agosto), Universidad de Antioquía, pp. 77-96.

BidART CAMPOS, Germán (1988): El constitucionalismo en las postrimerías del Siglo $X X$, (México, UNAM).

Biscarettidi Ruffia, Paolo (2000): Introducción al Derecho Constitucional Comparado. Las Formas de Estado y las Formas de Gobierno. Las Constituciones modernas. 1988-1990: un periodo de profundas transformaciones constitucionales en Occidente, en la URSS y en los Estados socialistas del Este europeo, (México, Fondo de Cultura Económica).

BuQueT, Daniel (2007): "Entre la legitimidad y la eficacia: Reformas en los sistemas de elección presidencial en América Latina", Revista Uruguaya de Ciencia Política, (Vol. 16, No 1, diciembre), Instituto de Ciencia Política, pp. 35-49. CAmeron, Maxwell (2010): "El Estado de la Democracia en los Andes, Revista de Ciencia Política, (Vol. 30, No 1), Instituto de Ciencia Política de la Pontificia Universidad Católica de Chile, pp. 5-20.

Cárdenas Gracia, Jaime (1994): Transición politica y reforma constitucional en México, (México, UNAM).

CAREY, John M. (2003): “The Reelection Debate in Latin America”, Latin American Politics and Society, (Vol. 45, No 1, abril), University of Miami, pp. 119-133. CARPIZO, Jorge (2007): Concepto de democracia y sistema de gobierno en América Latina, (México, UNAM).

CARPIZO, Jorge (2012): El presidencialismo mexicano, (México, Siglo XXI editores).

CHEREsky, Isidoro (2006): "Elecciones en América Latina. Poder presidencial y liderazgo político bajo la presión de la movilización de la opinión pública y la ciudadanía”, Nueva Sociedad, (No 206), pp. 14-26.

Colomer Vidal, Antonio (1992): "El presidencialismo iberoamericano", en AA. VV. Memorias del IV Congreso Iberoamericano de Derecho Constitucional, (México, UNAM), pp. 133 y 145. 
Corral, Margarita (2011): El estado de la democracia en América Latina: un análisis comparado de las actitudes de las elites y los ciudadanos, (Universidad de Salamanca, Boletín PNUD-Instituto de Iberoamérica).

Fix-Zamudio, Héctor y Valencia Carmona, Salvador (2011): Derecho Constitucional mexicano y comparado (México, Editorial Porrúa).

Giovanni, Sartori (1996): Ingeniería Constitucional Comparada, (México, Fondo de Cultura Económica).

Gross Espiell, Héctor (1978): Las Constituciones del Uruguay, (Madrid, Centro de Estudios Constitucionales).

Ratto Trabucco, Fabio (2005): "La experiencia constitucional del gobierno directorial o "colegiado" en "Uruguay", Cuadernos Constitucionales de la Cátedra Fadrique Furió Ceriol, (No 50/51), Universidad de Valencia, pp. 35-75.

LiNZ, Juan (1990, a): “Democracia: presidencialismo o parlamentarismo: ¿Hace alguna diferencia?", en Óscar Godoy (Ed.), Hacia una democracia moderna. La opción parlamentaria, (Santiago de Chile, Universidad Católica de Chile), pp. 41-108.

Linz, Juan (1990, b): "The Perils of Presidentialism", Journal of Democracy, (Vol. 1, No 1), The Johns Hopkins University Press, pp. 51-69.

Linz, Juan (1990, c): “The Virtues of Parliamentarism”, Journal of Democracy, (Vol. 1, No 4), The Johns Hopkins University Press, pp. 84-91.

Linz, Juan y Valenzuela Bowie, Arturo (2013): "Los peligros del presidencialismo", Revista Latinoamericana de política comparada, (Vol. 7, julio), Centro Latinoamericano de Estudios Políticos, pp. 11-31.

Levine, Daniel y Molina, José Enrique (2011): "Calidad de la democracia: fortalezas y debilidades en América Latina”, Revista Latinoamericana de Política Comparada, (Vol. 5, julio), Centro Latinoamericano de Estudios Políticos, pp. 95-113.

Loewenstein, Karl (1998): Teoría de la Constitución, (Traducc. Alfredo Gallego Anabitarte, Barcelona, Ariel).

Martínez Barahona, Elena y Brenes Barahona, Amelia (2012): "Y volver, volver, volver... Un análisis de los casos de intervención de las Cortes Supremas en la reelección presidencial en Centroamérica", Anuario de Estudios Centroamericanos, (Vol. 38), pp. 109-136.

MASSAL, Julie (2012): “El cambio constitucional ¿̨factor de democratización?”, Análisis Politico, (Vol. 27, No 75), Universidad Nacional de Colombia, pp. 29-45. 
Mainwaring, Scott; Bejarano, Ana María y Pizarro, Eduardo (Eds.). La crisis de la representación democrática en los países andinos, (Bogotá, Editorial Norma).

McConnell, Shelley A. (2010): "The Return of Continuismo?", Current History Journal, (Vol. 109, No 724, febrero), pp. 74-80.

Molina, José Enrique (2000): "Los sistemas electores de América Latina", Cuadernos Capel, (No 46), Costa Rica, Instituto Interamericano de Derechos Humanos-Centro de Asesoría y Promoción Electoral.

Negretto, Gabriel (2209): "Paradojas de la reforma constitucional en América Latina”, Journal of Democracy, (Vol.1, No 1), National Endowment for Democracy Johns Hopkins University, pp. 39-54.

Nogueira Alcalá, Humberto (2008): "Consideraciones sobre los tipos de gobierno presidencialista y semipresidencialista en la reforma constitucional", Boletín Mexicano de Derecho Comparado, (Número conmemorativo, sexagésimo aniversario, 1948-2008), UNAM, pp. 711-791.

Nohlen, Dieter, y Solari, Aldo (Comp.) (1988): Reforma politica y consolidación democrática. Europa y América Latina, (Caracas, Nueva Sociedad).

Nohlen, Dieter (1991, a): "Presidencialismo vs. parlamentarismo en América Latina. Notas sobre el debate actual desde una perspectiva comparada", Revista de Estudios Políticos, (No 74, octubre-diciembre, Nueva Época), pp. 43-54.

Nohlen, Dieter y Fernández, Mario (1991, b): (Eds.). Presidencialismo versus Parlamentarismo: América Latina, (Caracas, Nueva Sociedad).

NoHlen, Dieter (2012): "El Presidencialismo: análisis y diseños institucionales en su contexto", Revista Latinoamericana de política comparada, (No 6), Centro Latinoamericano de Estudios Políticos, pp. 49-75.

Nohlen, Dieter (2013): "Presidencialismo versus parlamentarismo. Dos enfoques contrapuestos", Revista Latinoamericana de política comparada, (Vol. 7, julio), Centro Latinoamericano de Estudios Políticos, pp. 61-76.

Orozco Henríquez, J. Jesús (2008): "Tendencias recientes en los sistemas presidenciales latinoamericanos", Boletín Mexicano de Derecho Comparado, (Número conmemorativo, sexagésimo aniversario, mayo-agosto), UNAM, pp. 793-858.

SÁNChez Agesta, Luis (1980): Curso de derecho constitucional comparado, 7a ed., (Madrid, Universidad Complutense de Madrid.

Serrafero, Mario D. (2011): "La reelección presidencial indefinida en América Latina”, Revista de Instituciones, Ideas y Mercados, (No 54, mayo), pp. 225-259.

Serrano, Felipe Victoriano (2010): "Estado, golpe de estado y militarización en América Latina: una reflexión histórico-política”, Argumentos, (Vol. 23, 
No 64, septiembre-diciembre 2010), Universidad Autónoma Metropolitana, pp. 175-183.

TREMINIO SÁNCHEZ, Ilka (2013): "Las reformas a la reelección presidencial en América Latina”, Estudios Sociológicos, (Vol. XXXI, No 91, enero-abril), pp. 59-85.

Treminio SÁncheZ, Ilka (2015): "Las reformas a la reelección presidencial del nuevo siglo en América Latina. Tres intentos de reforma y un golpe de estado", Politica y gobierno, (Vol. 22, No 1, enero-junio), pp. 147-173.

VAladés, Diego (1988). El control del poder en México, (México, UNAM).

VARGAS Cullell, Jorge (2011): "La calidad de la democracia y el estudio comparado de la democratización”, Revista Latinoamericana de Politica Comparada, (Vol. 5, julio), pp. 67-94.

Viciano Pastor, Roberto y Martínez Dalmau, Rubén (2011): "El nuevo constitucionalismo latinoamericano: fundamentos para una construcción doctrinal", Revista General de Derecho Público Comparado, (No 9), Instituto de Derecho Público, Universidad Rey Juan Carlos, pp. 1-24.

Viciano Pastor, Roberto y Martínez Dalmau, Rubén (2010): "Los procesos constituyentes latinoamericanos y el nuevo paradigma constitucional", IUS, (No 25, Nueva Época), Instituto de Ciencia Jurídica, pp. 7-29.

Villabella Armengol, Carlos (2014): "El Derecho Constitucional en Latinoamérica. Un cambio de paradigma”, en Roberto Viciano Pastor, Estudios sobre un nuevo constitucionalismo latinoamericano (España, Tirant lo Blanch), pp. 51-77.

Villabella Armengol, Carlos (2017): "El constitucionalismo contemporáneo de América Latina. Breve estudio comparado", Boletín Mexicano de Derecho Comparado, (Año XLX, No 149, mayo-agosto 2017), pp. 943-979. 